Paediatr. Paedolog. 2020 · 55:248-251 https://doi.org/10.1007/s00608-020-00813-1 Online publiziert: 9 . Juli 2020 (c) Der/die Autor(en) 2020

\section{J. Berger ${ }^{1} \cdot$ M. Neuberger ${ }^{1,2}$}

'Zentrum f. Public Health, Medizin. Univ. Wien, Wien, Österreich

${ }^{2}$ Kommission Klima \& Luftqualität, Österr. Akademie d. Wissenschaften, Wien, Österreich

\title{
Wer schützt Österreichs Kinder und Jugendliche vor Tabakindustrie und -handel?
}

In Teilen Europas beginnen noch immer untragbar viele Jugendliche mit dem Rauchen, und die Rauchanfänger werden immer jünger, weil das Marketing der Tabakindustrie erfolgreicher ist als die Tabakkontrolle. In Österreich gelang es erst am 1. November 2019 die rauchfreie Gastronomie einzuführen, die in Nordund Westeuropa schon seit 2004 von einer Reduktion der Frühgeburten sowie von einer Abnahme der Hospitalisierungen von Kindern und Jugendlichen wegen Asthma gefolgt war. Das Bewusstsein der Eltern und Erzieher zur Gefährlichkeit des Passivrauchens hatte in diesen Ländern zugenommen und in der Folge nahm Passiv- und Aktivrauchen von Kindern ab. Österreich hinkt dieser internationalen Entwicklung noch hinterher. Statistik Austria fand bei Jugendlichen hohe Raten von Passivrauchern und insgesamt einen deutlich höheren Raucheranteil als im OECD- oder EU-Schnitt.

In der ESPAD-Studie gaben $28 \%$ der befragten 15-jährigen österreichischen Schüler im Februar bis Juni 2019 an, in den vorangegangenen 30 Tagen entweder Tabakzigaretten, Wasserpfeife oder E-Zigaretten konsumiert zu haben, während die Monatsprävalenz von Tabakzigaretten bei $22 \%$ lag und $12 \%$ angaben, täglich Tabakzigaretten zu rauchen. Die Lebenszeitprävalenz des Konsums von Tabakzigaretten betrug $44 \%$, von Wasserpfeifen $47 \%$ und von E-Zigaretten $38 \%$ [1].

Der internationale Trend zur E-Zigarette als neuer Einstieg in die Nikotinsucht bekam einen Rückschlag, nachdem in der zweiten Jahreshälfte in den USA eine akute Dampferkrankheit (EVALI) zu über 2000 Hospitalisierungen geführt hatte, von denen drei Viertel jünger als 35 Jahre waren. Bis Februar 2020 starben 68 Erkrankte, meist mit bilateralen Pneumonien und ADHS. Nikotin, THC und Vitamin-E-Acetat bzw. das bei der Pyrolyse daraus entstehende Keten scheinen in vielen (allerdings nicht allen) Fällen beteiligt gewesen zu sein, was daran erinnert, dass auch bei uns im Internet Anleitungen $\mathrm{zu}$ finden sind, wie man durch „dripping“ illegale Drogen wie Cannabis mittels E-Zigaretten konsumieren kann.

Gemessen an der Kaufkraft sind Tabaksteuern und Zigarettenpreise in Österreich geringer als in Nachbarländern und es wird für Zigaretten in unzähligen Verkaufsstellen hemmungslos geworben, auch in deren Auslagen und auf Tausenden Automaten, die gern auf Schulwegen platziert werden. Schüler werden mit Waren für Kinder (Hefte, Sticker, Sweets, Softdrinks, sogar Spielzeug) in Trafiken gelockt und sind dort Tabakrauch und Werbung ausgesetzt. Für die Zielgruppe der Kinder werden Trafikanten von ihrer Branche auch über Süßwaren- und Spielzeugmessen informiert. Gratiszigaretten dürfen bei jeder neuen Sorte verteilt werden und die Alterskontrolle beim Zigarettenkauf wird durch keine unabhängige Stelle überwacht (wie das Glücksspiel), sondern durch die Monopolverwaltung, die nur am Umsatz der Trafiken interessiert ist. Zwar haben die Länder seit 01.01.2019 endlich das Schutzalter auf 18 Jahre angehoben, aber die Erkenntnis der Suchtforscher hat sich noch nicht herumgesprochen, dass man die Dealer bei Gesetzesverletzungen bestrafen und

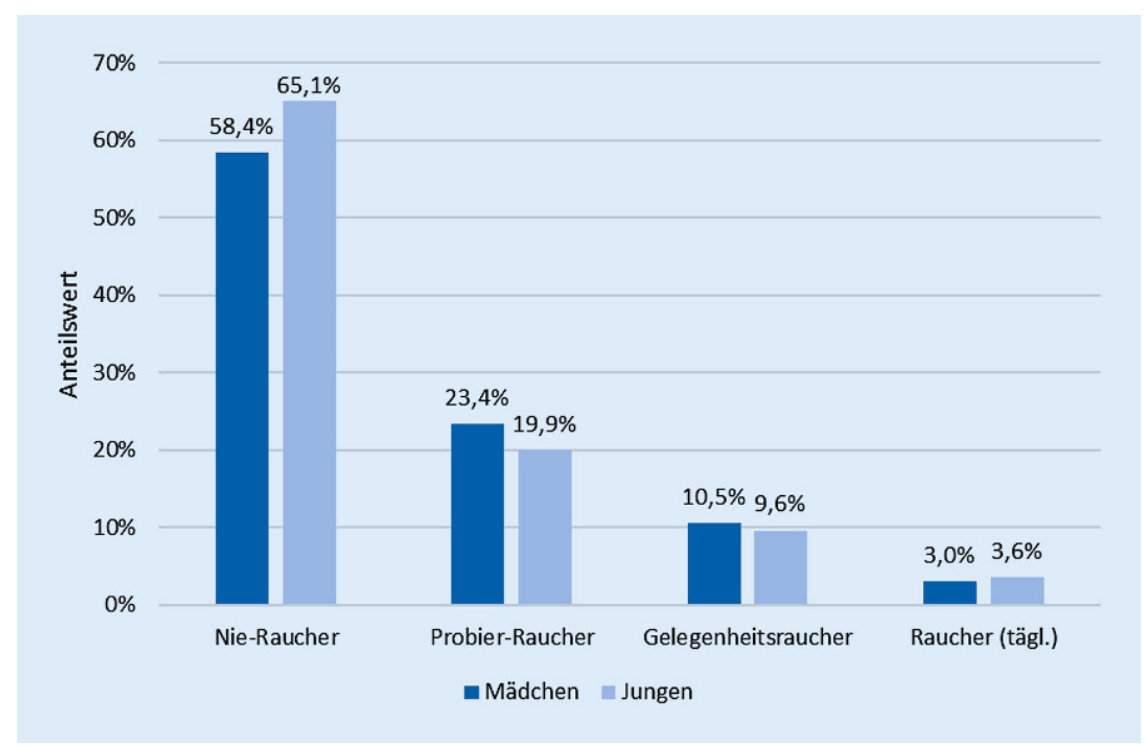

Abb. $1 \Delta$ Anteile von Nie-, Probier-, Gelegenheits- und täglichen Rauchern im Alter von 13 bis 16 Jahren 


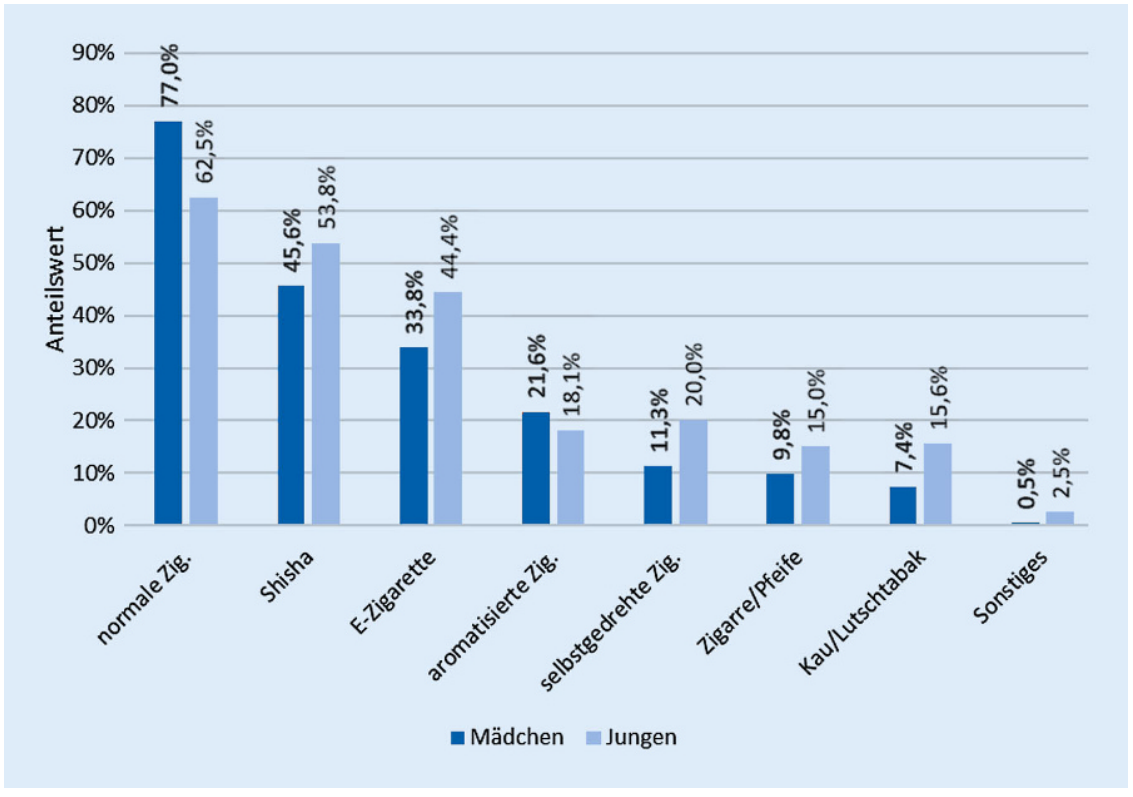

Abb. 2 \ Tabak- und Nikotinkonsum von 13- bis 16-jährigen Schülern und Schülerinnen

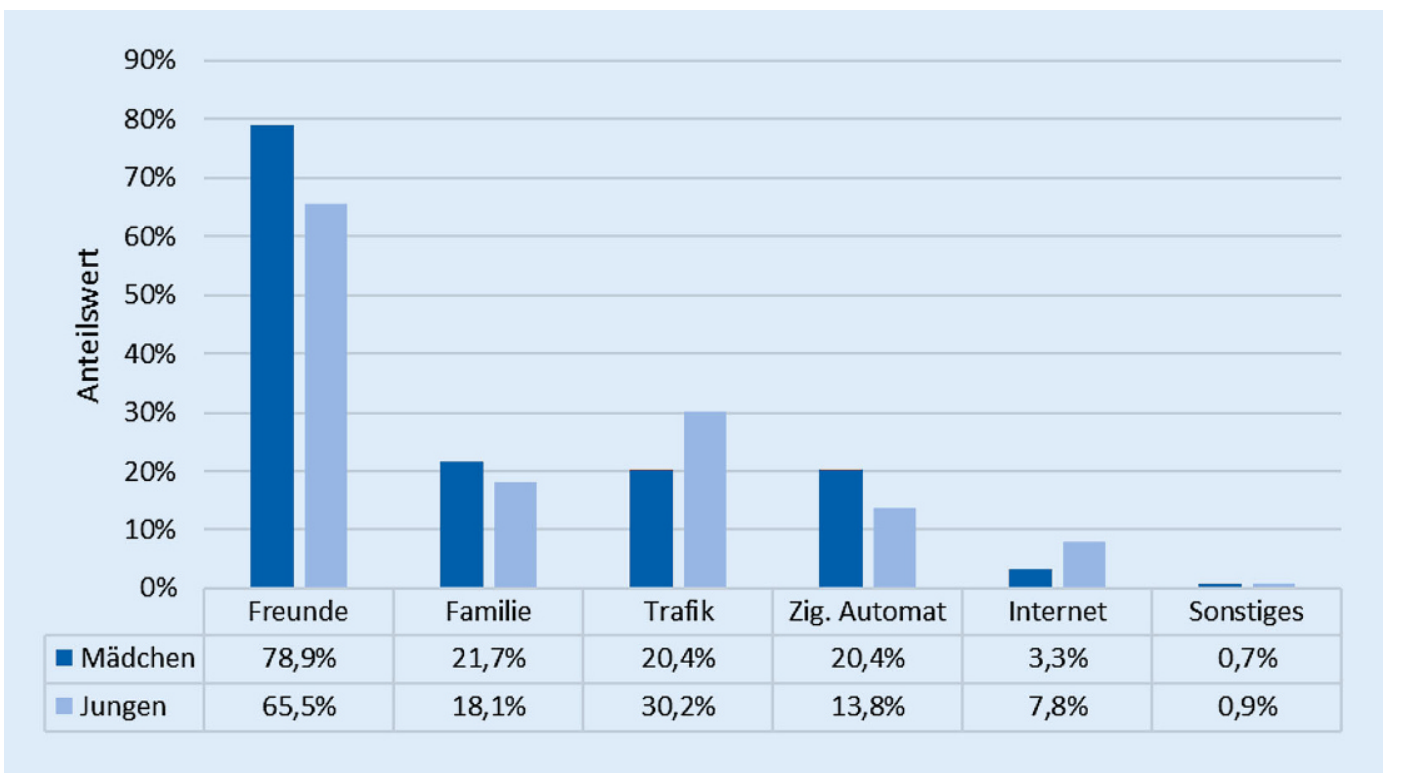

Abb. $3<$ Woher 13- bis 16-jährige Schüler und Schülerinnen in Österreich Tabak-und Nikotinprodukte beziehen

ihren jugendlichen Opfern helfen muss. In 4 Bundesländern wurden noch nicht einmal die gesetzlichen Voraussetzungen geschaffen, um Testkäufe durch Minderjährige in Trafiken, Supermärkten und bei Tankstellen durchführen zu können, was in westlichen Ländern längst Standard ist, mit Verdoppelung des Bußgelds bei Wiederholung der Gesetzesübertretung und schließlich Lizenzentzug, um Jugendliche vor Nikotinhändlern wirksam zu schützen [2].

Ziel unserer Studie (in Burgenland, Kärnten, Niederösterreich, Steiermark und Wien) war die Erfassung von Einstellung zum Rauchen und Raucherstatus von 13- bis 16-jährigen Schülern nach der Anhebung des gesetzlichen Schutzalters am 01.01.2019. Derzeit werden noch familiäre Einflüsse, schulische Einflüsse und Beziehungen zu Ernährung und Sport analysiert, die in 3 separaten Diplomarbeiten der Medizinischen Universität Wien bearbeitet werden. Die nachfolgenden Ergebnisse sind im Detail in der Diplomarbeit von Julia Berger dargestellt.

\section{Methoden}

In 9 Schultypen wurden im Herbst 2019 in zufällig ausgewählten Klassen für 13bis 16-Jährige $\mathrm{zu}$ Beginn einer Unterrichtsstunde Fragebögen an alle anwesenden Schüler verteilt. Von den 1082 durch Medizinstudenten ausgeteilten und anonym von den Schülern ausgefüllten Fragebögen mussten 53 (4,9\%) ausgeschieden werden, weil die Schüler jünger als 13 oder älter als 16 Jahre waren oder das Alter bzw. Geschlecht nicht ausgefüllt hatten. Von 1029Schülern entfielen auf Neue 
Mittelschulen (NMS) 15,4\%, Polytechnische Schulen (PTS) 14,9\%, Handelsschulen 5,9\%, Berufsschulen 4,4\%, Handelsakademien $29,6 \%$, Allgemeinbildende höhere Schulen (AHS) 9,2\%, Höhere Technische Lehranstalten (HTL) 7,9\%, Handelsakademien 29,6 \%, Höhere Lehranstalten für wirtschaftliche Berufe 9,5\% und eine Höhere Lehranstalt für Mode und Bekleidungstechnik 3,3\% [3].

Nach Antworten auf 2 Fragen zum Rauchverhalten wurden 4 Gruppen gebildet (•Tab. 1).

\section{Ergebnisse}

Die - Abb. 1 zeigt, dass 58,4\% der Mädchen und 65,1\% der Jungen (noch) keine Erfahrung mit Tabak hatten. Vom 13. bis zum 16. Lebensjahr nahmen die NieRaucher von 66 auf $58 \%$ ab.

Von 395 Fällen mit Tabakerfahrung gaben 381 (96,5\%) ihr Alter beim Erstkonsum an: 4, 5 und 6 Jahre wurden je einmal angegeben; 5,0\% hatten mit 11 Jahren, $11,8 \%$ mit $12,21,0 \%$ mit 13 , $27,6 \%$ mit $14,22,3 \%$ mit 15 und $4,5 \%$ mit 16 Jahren erstmals Tabak geraucht. Der Mittelwert lag bei 13,3 \pm 1,9 Jahren. Mädchen hatten mit $41,6 \%$ schon häufiger $(p=0,028)$ Rauchwaren probiert als Jungen (34,9\%), aber ihr Rauchbeginnalter unterschied sich nicht signifikant.

Am häufigsten wurden normale Tabakzigaretten konsumiert, besonders von Mädchen (-Abb. 2). An zweiter Stelle folgt die Wasserpfeife (Shisha). Jungen konsumieren signifikant häufiger selbstgedrehte Zigaretten, E-Zigaretten und Lutschtabak und weniger traditionelle Zigaretten als Mädchen.

Ebenfalls mit Mehrfachangaben wurden die in - Abb. 3 dargestellten Bezugsorte für Tabak- und Nikotinprodukte genannt. Am häufigsten bekamen die minderjährigen Raucher ihre Rauchwaren von Freunden, besonders die Mädchen. Schon an 2. Stelle stand die Trafik, wo 30,2\% der rauchenden Jungen ihre Rauchwaren kauften. Bei Mädchen waren Familie, Trafik und Zigarettenautomat von gleicher Bedeutung und die zweitwichtigsten Bezugsquellen. Die genannten Geschlechtsunterschiede waren signifikant. Internetkäufe spielen derzeit in Österreich noch eine gerin-

Paediatr. Paedolog. 2020 ·55:248-251 https://doi.org/10.1007/s00608-020-00813-1

(c) Der/die Autor(en) 2020

\section{J. Berger $\cdot$ M. Neuberger \\ Wer schützt Österreichs Kinder und Jugendliche vor Tabakindustrie und -handel?}

\section{Zusammenfassung}

Zum Weltnichtrauchertag 2020 ruft die WHO dazu auf, die Jugend vor der Manipulation durch die Tabak- bzw. Nikotinindustrie zu schützen. Bei der Tabakprävention haben Mittel- und Osteuropa Nachholbedarf. In Österreich wurde das Schutzalter erst 2019 auf 18 Jahre angehoben. Danach erhoben wir bei 13- bis 16-jährigen Schülern in 5 Bundesländern (Burgenland, Kärnten, Niederösterreich, Steiermark, Wien) Konsum und Bezugsquellen von Tabak- und Nikotinprodukten. Von $95 \%$ der Stichprobe erhielten wir auswertbare Antworten. Von 1029 Jugendliche gaben $38,4 \%$ zu, schon mit Rauchwaren Erfahrung zu haben (41,6\% der Mädchen und 34,9\% der Jungen), wobei Tabakzigaretten am häufigsten konsumiert wurden, gefolgt von Shisha und E-Zigaretten. Bezugsquellen sind meist Freunde, aber
Mädchen benutzen häufiger auch Automaten (11,6\%), meist zum Bezug normaler Zigaretten, während Jungen verschiedene Tabak- und Nikotinprodukte und das zu $30,2 \%$ in der Trafik kaufen. Im Vergleich zu früheren Studien ist der Anteil täglicher Raucher zwar auf 3,3\% (2,4-4,3\%) gesunken, aber der Anteil der Gelegenheitsraucher ist auf $10,1 \%(8,4-12,0 \%)$ gestiegen. Die Raucherprävalenz bei Jugendlichen liegt in Österreich noch immer über dem EUSchnitt. Dringend nötig wären Testkäufe zur Alterskontrolle in Trafiken, die Abschaffung der Zigarettenautomaten und die Förderung des Nichtrauchens.

\section{Schlüsselwörter}

Jugendschutz · Minderjährige · Nikotin ·

Zigaretten · Survey

\section{Who Protects Austrian Children and Adolescents from the Tobacco Industry and Trade?}

\section{Abstract}

On World No Tobacco Day 2020, the WHO called for the protection of youth from manipulation by the tobacco/nicotine industry. In tobacco prevention, Central and Eastern Europe are still lagging behind. In Austria, the legal age to buy tobacco was not raised from 16 to 18 years until 2019. Afterward, we surveyed the consumption and sources of tobacco and nicotine products of 13- to 16-year-old pupils in five Austrian states (Burgenland, Carinthia, Lower Austria, Styria, and Vienna). We received questionnaires from $95 \%$ of the sample. Of 1,029 adolescents, $38.4 \%$ admitted experience with tobacco or related products $(41.6 \%$ of the girls, $34.9 \%$ of the boys). Tobacco cigarettes were most frequently used, followed by shisha and e-cigarettes. Sources of supplies are mostly friends, but girls buy their tobacco products more frequently from vending machines (11.6\%) and mostly to source normal cigarettes, whereas boys buy various tobacco and nicotine products, $30.2 \%$ in tobacconists'. Compared with earlier studies, the proportion of daily smokers has decreased to $3.3 \%(2.4-4.3 \%)$, but the proportion of occasional smokers has increased to $10.1 \%$ (8.4-12.0\%). The prevalence of smoking in Austrian adolescents is still higher than the EU average. Mystery shopping in tobacconists' for age control, the banning of vending machines, and the promotion of non-smoking are urgently needed.

\section{Keywords}

Youth protection - Minors - Nicotine . Cigarettes · Survey ge Rolle, doch ist bemerkenswert, dass 12 von 14 Schülern, die Zigaretten im Internet kaufen, auch einen Konsum von E-Zigaretten angeben. Die dramatische Zunahme von E-Zigaretten wie in Nordamerika ist gottlob bei Jugendlichen in Österreich nicht zu sehen, aber mit Aromen versetzte Produkte, die auf den Geschmack von Kindern und Jugendlichen abgestimmt sind, könnten trotzdem noch ein Nikotinproblem der Zukunft werden [4]. Wünschenswert wäre die Streichung von Ausnahmen für Aromen und Verpackungen, die für Kinder und Jugendliche attraktiv sind. Nach den Ergebnissen der vorlie- 


\begin{tabular}{lll} 
Tab. 1 & Klassifizierung des Rauchverhaltens 13- bis 16-jähriger Schüler und Schülerinnen \\
\hline & Jemals Tabak geraucht? & Wie oft rauchst du derzeit? \\
\hline Nie-Raucher & Nein & - \\
\hline Probier-Raucher & Ja & Ich rauche nicht (mehr) \\
Gelegenheitsraucher & Ja & $\leq$ mehrmals pro Woche \\
Raucher & Ja & Täglich
\end{tabular}

genden Studie am dringendsten wären aber Testkäufe zur Alterskontrolle in Trafiken, die Abschaffung der Zigarettenautomaten, Verbote für Promotion, Sponsoring und Werbung, die auch den Verkaufsort einschließen, eine Erhöhung der Tabaksteuer mit Zweckbindung für Tabakprävention, die Denormalisierung des Rauchens in der Öffentlichkeit und die Förderung des Nichtrauchens. Rauchenden Kindern sollte geholfen und nur die Händler sollten bestraft werden. Seit 20. Mai 2019 lässt sich von jeder Zigarettenpackung (die man Minderjährigen abnimmt) ablesen, aus welcher Trafik sie stammt [5].

\section{Fazit für die Praxis}

Erfreulich ist ein leicht abnehmender Trend bei täglich rauchenden Jugendlichen, aber im Vergleich zu früheren Studien war bei Gelegenheitsrauchern eine Zunahme zu beobachten. Dieses Partyrauchen bereitet häufig den Einstieg ins regelmäßige Rauchen vor. Seit 01.11.2019 kann unsere Jugend zwar nicht mehr in Lokalen zum Rauchen und Shisha-Konsum verführt werden und auch Kinder bekommen dort keinen Anschauungsunterricht mehr im Rauchen und Dampfen; trotz Anhebung des Schutzalters von 16 auf 18 Jahre seit 01.01.2019 ist der Jugendschutz vor dem Tabakhandel in Österreich jedoch noch unbefriedigend. Bestraft werden sollten Händler, die Minderjährigen Nikotinprodukte verkaufen und den rauchenden Kindern sollte geholfen werden. Von Zigarettenpackungen, die man ihnen abnimmt, kann schon seit 20.05.2019 abgelesen werden, aus welcher Trafik sie stammen.
Funding. Open access funding provided by Medical University of Vienna.

\section{Einhaltung ethischer Richtlinien}

Interessenkonflikt. J. Berger und M. Neuberger geben an, dass kein Interessenkonflikt besteht.

Für diese Studie wurden von den Autoren Prüfungen und Bewilligungen der Bildungsdirektionen aller 5 Bundesländer sowie der Ethikkommission der Medizinischen Universität Wien (EKNr: 1736/2019) eingeholt.

Open Access. Dieser Artikel wird unter der Creative veröffentlicht, welche die Nutzung, Vervielfältigung Bearbeitung, Verbreitung und Wiedergabe in jeglichem Medium und Format erlaubt, sofern Sie den/die ursprünglichen Autor(en) und die Quelle ordnungsgemäß nennen, einen Link zur Creative Commons Lizenz beifügen und angeben, ob Änderungen vorgenommen wurden.

Die in diesem Artikel enthaltenen Bilder und sonstiges Drittmaterial unterliegen ebenfalls der genannten Creative Commons Lizenz, sofern sich aus der Abbildungslegende nichts anderes ergibt. Sofern das betreffende Material nicht unter der genannten Creative Commons Lizenz steht und die betreffende Handlung nicht nach gesetzlichen Vorschriften erlaubt ist, ist für die oben aufgeführten Weiterverwendungen des Materials die Einwilligung des jeweiligen Rechteinhabers einzuholen.

Weitere Details zur Lizenz entnehmen Sie bitte der Lizenzinformation auf http://creativecommons.org/ licenses/by/4.0/deed.de.

\section{Literatur}

1. https://www.sozialministerium.at/Themen/ Gesundheit/Drogen-und-Sucht/Berichteund-Statistiken/Europ\%C3\%A4ische-Sch \%C3\%BClerstudie-zu-Alkohol-und-anderenDrogen-.html.Zugegriffen: 6.7.2020

2. https://www.aerzteinitiative.at/_Kinder_1.htm. Zugegriffen: 6.7.2020 Commons Namensnennung 4.0 International Lizenz
3. Berger J, Neuberger M (2020) Occupational and environmental health benefits of smoking ban not yet arrived in Austrian youth. Int J Occup Environ Med. https://openaccesspub.org/ijoe/ article/1270

4. Shah SI, Javier JR, Brumberg HL (2020) The vapes of wrath: advocating to protect children from electronic nicotine systems in the age of flavored vapes. Pediatr Res 87:972-975. https://www. nature.com/articles/s41390-020-0872-z

5. https://ec.europa.eu/health/tobacco/tracking_ tracing_system_en.Zugegriffen:6.7.2020

Hinweis des Verlags. Der Verlag bleibt in Hinblick auf geografische Zuordnungen und Gebietsbezeichnungen in veröffentlichten Karten und Institutsadressen neutral. 